\title{
MS imaging and mass spectrometric synaptosome profiling identify PEP-19/pcp4 as a synaptic molecule involved in spatial learning in mice
}

\author{
Jeroen Aerts a,1, Annelies Laeremans ${ }^{\mathrm{a}, 1}$, Laurens Minerva a ${ }^{\mathrm{a}}$, Kurt Boonen ${ }^{\mathrm{b}}$, Budamgunta Harshavardhan ${ }^{\mathrm{c}}$, \\ Rudi D’hooge ${ }^{\mathrm{d}}$, Dirk Valkenborg ${ }^{\mathrm{c}, \mathrm{e}}$, Geert Baggerman ${ }^{\mathrm{c}, \mathrm{e}}$, Lutgarde Arckens ${ }^{\mathrm{a}, *}$ \\ a KU Leuven, Department of Biology, Laboratory of Neuroplasticity and Neuroproteomics, Naamsestraat 59, 3000 Leuven, Belgium \\ ${ }^{\mathrm{b}}$ KU Leuven, Department of Biology, Laboratory of Functional Genomics and Proteomics, Naamsestraat 59, 3000 Leuven, Belgium \\ c Center for Proteomics, UAntwerp, Antwerp, Belgium \\ d KU Leuven, Laboratory of Biological Psychology, Tiensestraat 102, 3000 Leuven, Belgium \\ e Unit Environmental Risk \& Health, VITO, Mol, Belgium
}

\section{A R T I C L E I N F O}

\section{Article history:}

Received 31 May 2016

Received in revised form 7 October 2016

Accepted 11 October 2016

Available online $\mathrm{xxxx}$

\section{Keywords:}

HPLC

MALDI

Mouse

Striatum

Synaptosome

\begin{abstract}
A B S T R A C T
The Morris water maze (MWM) spatial learning task has been demonstrated to involve a cognitive switch of action control to serve the transition from an early towards a late learning phase. However, the molecular mechanisms governing this switch are largely unknown. We employed MALDI MS imaging (MSI) to screen for changes in expression of small proteins in brain structures implicated in the different learning phases. We compared mice trained for 3 days and 30 days in the MWM, reflecting an early and a late learning phase in relation to the acquisition of a spatial learning task. An ion with $\mathrm{m} / \mathrm{z}$ of 6724 , identified as PEP-19/pcp4 by top-down tandem MS, was detected at higher intensity in the dorsal striatum of the late learning phase group compared with the early learning phase group. In addition, mass spectrometric analysis of synaptosomes confirmed the presence of PEP-19/pcp4 at the synapse. PEP-19/pcp4 has previously been identified as a critical determinant of synaptic plasticity in locomotor learning. Our findings extend PEP-19/pcp4 function to spatial learning in the forebrain and put MSI forward as a valid and unbiased research strategy for the discovery and identification of the molecular machinery involved in learning, memory and synaptic plasticity.
\end{abstract}

(c) 2016 Elsevier B.V. All rights reserved.

\section{Introduction}

The hidden-platform Morris water maze (MWM) is a visually guided spatial navigation task in which a platform location is learned in relation to distal environmental cues [1] and this learning paradigm is typically characterized by the transition from an early learning phase towards a late learning phase in both rodents and humans [2,3]. In the early learning phase, navigation is driven by flexible goal-directed actions whereas the late learning phase exhibits rigid habit formation which is induced by overtraining [4-6]. The switch between goal-directed behavior and habitual actions occurs within the cortico-striatal loop with a prominent role for the dorsal striatum that receives extensive topographically organized afferent projections from higher cortical areas [4]. Indeed, previous research revealed that in the early learning phase the

\footnotetext{
* Corresponding author at: Laboratory of Neuroplasticity and Neuroproteomics, KU Leuven, Naamsestraat 59, box 2467, 3000 Leuven, Belgium.

E-mail address: Lut.Arckens@kuleuven.be (L. Arckens).

1 Shared first
}

navigation is orchestrated by both place cells in the hippocampus that form a cognitive map of the environment, and the dorsomedial striatum (DMS) in which action-outcome associations are encoded [2]. In contrast, habitual behavior in the late learning phase is driven by stimulus-response associations that are consolidated and encoded in the dorsolateral striatum (DLS) $[1,4,7,8]$. Both goal-directed and habitual navigation strategies are engaged in parallel during the learning process but the DMS and DLS develop strikingly different strategy-specific neuronal activity patterns $[2,3,7,9]$, demonstrating that the dorsal striatum is an important node to direct action control.

However, the underlying molecular mechanisms driving this switch in action control are largely unknown. To this end MALDI MS imaging (MSI) is a powerful discovery tool that provides both spatial and chemical information without a priori knowledge of endogenous biomolecules and with the strength of screening hundreds of analytes simultaneously in a single tissue section $[4-6,10,11]$. In this study, we employed MSI to elucidate potentially new, region-specific molecular determinants governing cognitive action control by comparing the expression patterns of small proteins in the mouse striatum between 
3 days (early learning phase) and 30 days (late learning phase) of training in the MWM.

\section{Material and methods}

\subsection{Animal training and tissue preparation}

All experiments were performed on eight-week-old female C57Bl/6 J mice (Janvier Elevage, Le Genest-St-Isle, France), which were housed under standard laboratory conditions under a $12 \mathrm{~h}$ dark/light cycle with food and water available ad libitum. All experiments were approved by the Ethical Research Committee of KU Leuven and were in strict accordance with the European Communities Council Directive of 22 September 2010 (2010/63/EU).

Morris water maze training was performed as described in detail in Woolley et al. [2]. Briefly, experimental mice were trained to find the hidden platform either for 3 days ( $3 \mathrm{~d}$, one session of four trials per day) or 30 days ( $30 \mathrm{~d}$, two sessions of four trials per day for the first 25 days of training, then one session of four trials per day for the remaining five days; five consecutive training days were followed by two rest days). At the start of each trial the mouse was placed at the edge of the pool facing toward the center at one of four starting locations. During all trials the experimenter remained seated at a fixed location. When a trial was not completed in two minutes the mouse was guided to the platform and remained there for $15 \mathrm{~s}$. Overall task performance was evaluated by calculating the time taken to find the hidden platform (escape latency) and spatial performance was determined as the average distance between the mouse and the hidden platform. During the early learning phase, a steep reduction in escape latency was observed (Fig. 1). After three days, further training of the $30 \mathrm{~d}$ group resulted in additional large performance gains followed by a prolonged habituation phase where a continued level of high performance was achieved. The reduction in escape latency was accompanied by an enhanced goal-directed focus towards the platform.

After training, animals were killed by cervical dislocation, brains rapidly removed and immediately frozen in 2-methylbutane (Merck, Overijse, Belgium) at a temperature of $-40{ }^{\circ} \mathrm{C}$ and stored at $-80{ }^{\circ} \mathrm{C}$ until sectioning.

\subsection{MALDI MS imaging}

\subsubsection{Sample preparation}

Coronal sections of $14 \mu \mathrm{m}$ from +1.10 to $0.38 \mathrm{~mm}$ relative to Bregma were cut on a cryostat (Microm HM 500 OM, Walldorf, Germany), collected on conductive ITO-coated glass slides (Bruker Daltonick $\mathrm{GmbH}$, Bremen, Germany) and lyophilized for $20 \mathrm{~min}$. Sections from $3 \mathrm{~d}$ and $30 \mathrm{~d}$ trained animals ( $\mathrm{n}=2$ /condition) were collected on the same ITO slide and prepared pairwise to minimize technical variability. Next, tissue sections were rinsed successively in 70\% ethanol (30 s), $100 \%$ ethanol (30 s), 100\% chloroform (dipped 10 times) and water (dipped 2 times), after which the sections were dried using a nitrogen gas flow. The MALDI matrix solution consisted of $50 \mathrm{mg} / \mathrm{ml} \mathrm{DHB}$ (Acros Organic, Geel, Belgium) in 50\% methanol (Sigma-Aldrich, Steinheim, Germany) with 0.1\% TFA (Sigma-Aldrich, Steinheim, Germany) and was uniformly applied over the tissue slices by spray coating (Master G Series Airbrush kit) two consecutive layers in a raster pattern with 1 min drying time in between layers.

\subsubsection{Instrumentation}

All MALDI measurements were conducted on an Ultraflex II instrument equipped with a smartbeam-laser and FlexImaging 2.1 and ClinProTools 2.2 software packages by Bruker Daltonik $\mathrm{GmbH}$ (Bremen, Germany). Spectra were acquired in positive linear mode in a mass range of 5000-20,000 Da with a sampling rate of $1 \mathrm{GS} / \mathrm{s}$. Each spectrum was the result of 200 consecutive shots at $100 \mathrm{~Hz}$. The lateral resolution was set to $65 \mu \mathrm{m}$ and all MSI runs were externally calibrated using a MALDI preparation of a standard protein mixture (Protein Calibration Standard I, Bruker Daltonik $\mathrm{GmbH}$ ) adjacent to each tissue section.

\subsubsection{Data analysis}

All spectra were preprocessed in FlexAnalysis using a smoothing and baseline subtraction routine. A Savitsky Golay algorithm was used for mass spectral smoothing (width 2 Da for 4 cycles) and a Top Hat baseline algorithm for baseline subtraction. Selected ion images were generated in FlexImaging and spectra from the regions of interest (ROIs) were extracted from the 3 days and 30 days experimental conditions and processed further in ClinProTools. All spectra were normalized to their TIC and peak of interest $(\mathrm{S} / \mathrm{N}>3)$ intensity ratios for 30 days to 3 days were calculated.

\subsection{Histology}

After MS measurements the tissue sections were Nissl-counterstained (Cresyl violet $1 \%$, Fluka Chemika, Sigma-Aldrich) according to standard procedures. Stained sections were compared to a stereotaxic mouse brain atlas [13] to allow accurate localization of anatomical landmarks and delineation of brain regions. Images of the stained coronal sections were obtained with a light microscope (Zeiss Axio Imager Z1) equipped

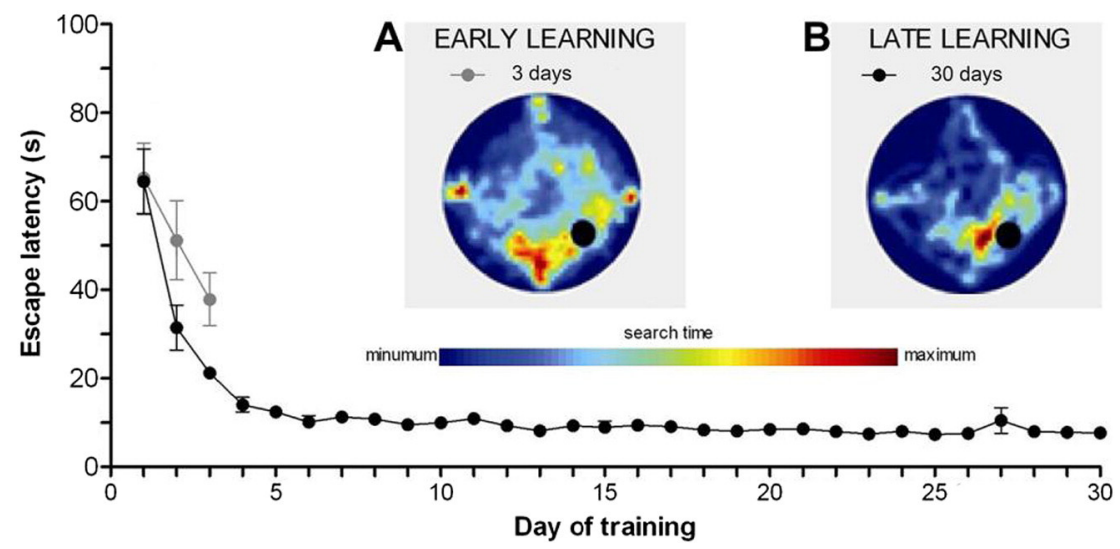

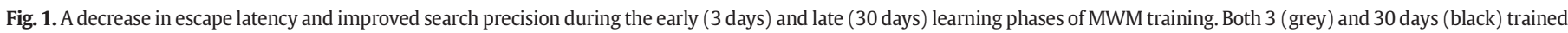

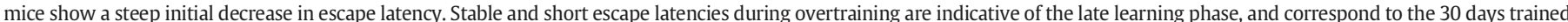

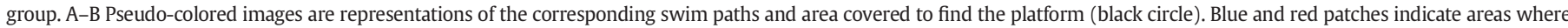

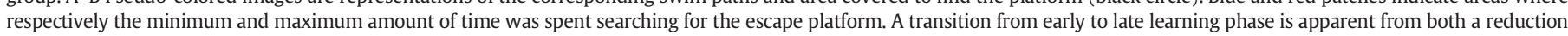
in escape latency as well as a reduced search area. Error bars represent SEM. 
with an AxioCam MRc 5 camera using the software program Axiovision Rel. 4.6 (Carl Zeiss and Benelux, Zaventem, Belgium).

\subsection{Protein identification}

To identify the peptides and proteins observed in the MALDI imaging experiment, extracts of mouse striatum samples were further analyzed by LC MS/MS. The striatal tissue was dissected as quickly as possible and immediately placed in ice cold peptide extraction solvent consisting of methanol:water:acetic acid (90:9:1; v:v:v). The sample was kept on ice and sonicated using a bar sonicator (Branson Sonifier SLPe cell disruptor) prior to centrifugation. The supernatant was collected from which the methanol was evaporated by using a vacuum centrifuge concentrator (Eppendorf 5301 concentrator centrifugal evaporator). Lipids were removed from the remaining aqueous residue, containing the peptides, by re-extraction with ethyl acetate and n-hexane. The aqueous solution was subsequently desalted by solid phase extraction using a Pierce C18 Spin Column (Thermo scientific) according to the manufacturers procedure. The obtained sample was again dried using the vacuum centrifuge concentrator and stored at $-20{ }^{\circ} \mathrm{C}$ prior to analysis.

\subsubsection{UPLC fractionation}

The prepared extract was fractionated offline with an Acquity UPLC system (Waters). The sample was reconstituted with $30 \mu \mathrm{l}$ of a $5 \%$ acetonitrile (ACN), $0.1 \%$ formic acid (FA) solution. The samples were loaded onto an X-bridge BEH130 C18 column with following dimensions: $150 \mathrm{~mm} \times 4.6 \mathrm{~mm}$ and $5 \mu \mathrm{m}$ particles (Waters). The peptides were eluted with a flow rate of $1.5 \mathrm{ml} / \mathrm{min}$ with a linear gradient of $2 \%$ solvent $\mathrm{A}$ ( $2 \% \mathrm{ACN}, 0.1 \%$ aqueous trifluoroacetic acid solution) into $60 \%$ solvent $\mathrm{B}$ ( $95 \% \mathrm{ACN}, 0.1 \%$ aqueous trifluoroacetic acid solution) over $10 \mathrm{~min}$. The peptide elution was monitored by measuring the absorbance at $\lambda=214 \mathrm{~nm}$ with the photodiode array and fractions were collected at 0.5 minute intervals. From each of the resulting fractions $1 / 20$ of the volume was transferred to a separate vial for MALDI-TOF analysis. All fractions were dried in a vacuum centrifuge and stored at $-20{ }^{\circ} \mathrm{C}$ until further analysis.

\subsubsection{MALDI-TOF analysis}

The aliquots were reconstituted in $5 \mu \mathrm{l}$ of $5 \% \mathrm{Acn}, 0.1 \%$ aqueous trifluoroacetic acid solution. $1 \mu \mathrm{l}$ of the each sample was applied to a target plate (stainless steel) and $0.5 \mu \mathrm{l}$ of a saturated matrix ( $\alpha$-cyano4-hydroxy-cinnamic acid) solution in 30\% acetonitrile aqueous solution was added and dried to air. Samples were analyzed using an AB4800 proteomics analyzer (Applied Biosystems) by MALDI-TOF (reflectron mode; $25 \times 20$ laser shots per spot; mass-range: 1000-10,000 Da; laser intensity: 2500).

\subsubsection{LC-MS/MS analysis}

Liquid chromatography mass spectrometric analysis was performed on an EasynLC 1000 nanoUPLC system connected to a Q-exactive plus Orbitrap mass spectrometer (all Thermo Scientific). The dried fraction(s) containing the peptide of interest were dissolved in $30 \mu \mathrm{l}$ of $2 \%$ acetonitrile in HPLC-grade water, $0.1 \%$ of formic acid. $10 \mu$ of the sample was loaded on the trapping column (Pepmap C18 RSLC $75 \mu \mathrm{m} \times 20 \mathrm{~mm}$, Thermo Scientific) with an isocratic flow of $2 \%$ acetonitrile in water with $0.1 \%$ formic acid at a flow rate adapted automatically to a maximum pressure of 780 Bar. After equilibration, the column-switching valve was switched, placing the pre-column online with the analytical capillary column, a Pepmap C18 RSLC, $2 \mu \mathrm{m}$, $50 \mu \mathrm{m} \times 150 \mathrm{~mm}$ nano column (Thermo Scientific). Separation was conducted using a linear gradient from $2 \%$ acetonitrile in water, $0.1 \%$ formic acid to $50 \%$ acetonitrile in water, $0.1 \%$ formic acid in $50 \mathrm{~min}$. The flow rate was set at $300 \mathrm{nl} / \mathrm{min}$. The mass spectrometer was set up in a data dependent MS/MS mode where a full scan spectrum (350-1850 m/z, resolution 70,000, max injection time; $100 \mathrm{~ms}$, AGC target was 3e6) was followed by a maximum of ten HCD tandem mass spectra (200-2000 m/z, resolution 35,000, max injection time; $200 \mathrm{~ms}$, AGC target was 1e5). Peptide ions were selected as the 10 most intense peaks of the MS1 scan. The normalized collision energy used was 35\% in HCD. We applied a dynamic exclusion list of $15 \mathrm{~s}$. An inclusion list was used, so that whenever the appropriate ions were detected in MS1 these would be selected for fragmentation with priority over other possible precursors regardless of their signal intensity.

\subsubsection{Data-analysis}

Fragmentation spectra of ions with $\mathrm{m} / \mathrm{z}$ predefined in the inclusion list were individually deconvoluted using Xtract algorithm in Xcalibur (Thermo Scientific) and fragment masses and intensities were exported as CSV files. Manual de novo sequencing was performed on the deconvoluted spectra to determine (a) sequence $\operatorname{tag}(\mathrm{s})$. The sequences obtained were searched in the Uniprot murine database using BLAST algorithm (http://blast.ncbi.nlm.nih.gov/) corrected automatically for short sequences. Prosight lite (http://prosightlite.northwestern.edu/) [14] was used to annotate the amino acid sequences of the entire corresponding proteins onto the deconvoluted fragmentation spectrum and to aid in the determination of the post-translational modifications. Settings were $10 \mathrm{ppm}$ mass accuracy for the fragmentation spectra and $1 \mathrm{Da}$ for the parent mass.

\subsection{Immunohistochemistry}

Brains of 3d ( $\mathrm{n}=2)$ and $30 \mathrm{~d}(\mathrm{n}=2)$ trained mice were rapidly removed and immediately frozen in 2-methylbutane (Merck, Overijse, Belgium) at a temperature of $-40{ }^{\circ} \mathrm{C}$ and stored at $-80{ }^{\circ} \mathrm{C}$ until sectioning. Ten- $\mu \mathrm{m}$-thick sections were prepared on a cryostat (Microm HM 500 OM, Walldorf, Germany), mounted on $0.1 \%$ poly-L-lysine (Sigma-Aldrich, St. Louis, MO)-coated slides and stored at $-80{ }^{\circ} \mathrm{C}$ until further processing. An indirect labeling with signal amplification was used to visualize PEP19/pcp4 in brain sections. Sections were incubated with a $0.3 \% \mathrm{H}_{2} \mathrm{O}_{2}$ - in Tris-buffered saline (TBS; $0.01 \mathrm{M}$ Tris, $0.9 \%$ $\mathrm{NaCl}, 0.3 \%$ Triton-X 100, pH 7.6). After pre-incubation with Pre Immune Goat serum (1:5 for $45 \mathrm{~min}$ ), the sections were incubated overnight with the primary PEP19/pcp4 antibody (1:10,000, gift from Prof. Dr. James I. Morgan). The following day, detection was performed using a biotinylated goat anti-rabbit immunoglobulin G (1:500, 30 min, Dako, Glostrup, Denmark) and an avidin-biotin-horseradish peroxidase solution (Vectastain Elite ABC, Vector Laboratories, Burlingame, CA). All incubations and rinses $(3 \times 10 \mathrm{~min})$ were performed in TBS. The sections were immunostained using the glucose oxidasediaminobenzidine-nickel method resulting in a black staining $[15,16]$. Finally, the sections were air dried, dehydrated, cleared with xylene, coverslipped and viewed under a Zeiss Axio-Imager equipped with a Zeiss Axiocam (Carl Zeiss Benelux). Images were taken with the ZEN software (Carl Zeiss Benelux).

\subsection{Quantitative in situ hybridization}

In situ hybridization (ISH) on brains of $3 \mathrm{~d}(\mathrm{n}=4)$ and $30 \mathrm{~d}(\mathrm{n}=5)$ trained mice was performed for PEP-19/pcp4 with a mouse-specific synthetic oligonucleotide probe (Eurogentec, Seraing, Belgium) with sequence $5^{\prime}$-ctcctgacgtcttgtcttttccgttggtcgctccggcactttgtc- $3^{\prime}$. As described previously [17-20], the probe was $3^{\prime}$-end labeled with ${ }^{33} \mathrm{P}$-dATP using terminal deoxynucleotidyl transferase (Invitrogen, Paisley, UK). Unincorporated nucleotides were separated from the labeled probe with miniQuick Spin ${ }^{\mathrm{TM}}$ Oligo Columns (Roche Diagnostics, Brussels, Belgium). Series of cryostat sections throughout the striatum were fixed, dehydrated and delipidated. The radioactively labeled probe was added to a hybridization cocktail ( $50 \%$ ( $\mathrm{vol} / \mathrm{vol}$ ) formamide, $4 \times$ standard saline sodium citrate buffer, $1 \times$ Denhardt's solution, $10 \%$ (wt/vol) dextran sulphate, $100 \mu \mathrm{g} / \mathrm{ml}$ herring sperm DNA, $250 \mu \mathrm{g} / \mathrm{ml}$ tRNA, $60 \mathrm{mM}$ dithiothreitol, 1\% (wt/vol) $N$-lauroyl sarcosine, $20 \mathrm{mM}$ 
$\left.\mathrm{NaHPO}_{4}, \mathrm{pH} 7.4\right)$ and applied to a series of cryostat sections $\left(10^{6} \mathrm{cpm}\right.$ per section) for an overnight incubation at $37^{\circ} \mathrm{C}$ in a humid chamber. The following day, sections were rinsed in $1 \times$ standard saline sodium citrate buffer at a temperature of $42{ }^{\circ} \mathrm{C}$, dehydrated, air-dried and exposed to an autoradiographic film (Biomax MR, Kodak, Zaventem, Belgium) together with a [14C] microscale (GE Healthcare). Films were developed in Kodak D19 developing solution after three weeks, followed by fixation in Rapid fixer (Ilford Hypam, Kodak). For each mouse, autoradiographic images were scanned at $1200 \mathrm{dpi}$ (CanoScan LIDE 600F, Canon, USA) and relative quantities of PEP-19/pcp 4 mRNA expression (mean gray value per pixel) were determined with ImageJ (Image processing and analysis in Java, National Institutes of Health). Optical density of the striatum was measured in three brain sections per mouse (3d: $n=4 ; 30 \mathrm{~d}: \mathrm{n}=5$ ) along the rostrocaudal axis (between $+1.10 \mathrm{~mm}$ and $+0.38 \mathrm{~mm}$ relative to Bregma). Mean gray values were averaged across hemispheres and brain slices, resulting in a single data point per animal. A normal distribution was verified using the Kolmogorov-Smirnov test and parallel equal variance between groups was tested. To compare PEP-19/pcp4 expression between the $3 \mathrm{~d}$ and $30 \mathrm{~d}$ condition a Mann-Whitney $\mathrm{U}$ rank-sum test for pairwise comparison of independent samples was applied with an $\alpha$-level set to 0.05 .

\subsection{Synaptosome analysis}

\subsubsection{Synaptosome preparation}

Synaptosomes were purified according to the protocol of Wishart et al. [21]. Two-month-old C57Bl/6 mice were obtained from the breeding facility of the KU Leuven. Mice were killed by cervical dislocation and the brains were rapidly removed. Complete brains $(n=8)$ were homogenized in an ice-cold isotonic sucrose solution (0.32 M sucrose, $1 \mathrm{mM}$ EDTA, $5 \mathrm{mM}$ Tris- $\mathrm{HCl}, \mathrm{pH}$ 7.4) and centrifuged at $900 \mathrm{~g}$ for $10 \mathrm{~min}$. The supernatant (S1) was collected and the pellet (P1) was resuspended in sucrose solution and centrifuged again at $900 \mathrm{~g}$ for $10 \mathrm{~min}$. The two supernatants were combined and centrifuged at $20,000 \mathrm{~g}$ for $15 \mathrm{~min}$. The resulting pellet containing synaptosomes was washed in a Krebs-like buffer $(118.5 \mathrm{mM} \mathrm{NaCl}, 4.7 \mathrm{mM} \mathrm{KCl}, 1.18 \mathrm{mM} \mathrm{MgCl}$, $0.1 \mathrm{mM}$ K2HPO4, $20 \mathrm{mM}$ Hepes, $1.3 \mathrm{mM} \mathrm{CaCl}$, $10 \mathrm{mM}$ glucose, $\mathrm{pH}$ 7.4 ) and centrifuged at $14,000 \mathrm{~g}$ for $10 \mathrm{~min}$. The synaptosomes were stored at $-80{ }^{\circ} \mathrm{C}$ until extraction.

\subsubsection{Identification of PEP-19/pcp4 in synaptosomes}

Chilled extraction solvent (methanol/water/formic acid, 90/9/1, v/v/ v) was added to the pellet and the sample was sonicated 5 times for $10 \mathrm{~s}$ using a bar sonicator. The extract was centrifuged at $20,000 \mathrm{~g}$ for $15 \mathrm{~min}$. The supernatant was delipadated using hexane and purified with HLB cartridges according to manufacturer's protocol (Waters, Milfor, MA). The samples were lyophilized and resuspended in $18 \mu$ of a $2 \%$ acetonitrile $0.1 \%$ formic acid solution and analyzed on a Q Exactive Orbitrap mass spectrometer (Thermo Scientific, San Jose, CA), coupled online to an Ultimate 3000 ultra-high performance liquid chromatography (UHPLC) instrument (Thermo Scientific, San Jose, CA) with an EasySpray source (ESI). The instrument was operated in positive ionization mode. The UHPLC system has a $2 \mu \mathrm{m}$ particle size, $100 \AA$ A pore size EASY-Spray Pepmap RSLC C18 column with dimensions $50 \mu \mathrm{m} \times$ $15 \mathrm{~cm}$ (Thermo Scientific, San Jose, CA) and a $3 \mu \mathrm{m}$ particle size, $100 \AA$ pore size, nanoviper, Acclaim Pepmap 100 C18 precolumn with dimensions $75 \mu \mathrm{m} \times 2 \mathrm{~cm}$ (Thermo Scientific, San Jose, CA). A sample volume of $8 \mu \mathrm{l}$ was injected. Buffer A consisted of $0.1 \%$ formic acid in water and buffer B of $80 \%$ acetonitrile and $0.08 \%$ formic acid. After 10 min equilibration, buffer B increased from $4 \%$ to $10 \%$ in $10 \mathrm{~min}, 10 \%$ to $25 \%$ in $150 \mathrm{~min}, 25 \%$ to $45 \%$ in $50 \mathrm{~min}$ and a steep increase to $95 \%$ in $5 \mathrm{~min}$. The flow rate was $300 \mathrm{nl} / \mathrm{min}$. The mass spectrometer operated in data dependent mode and all MS1 spectra were acquired in the positive ionization mode with an $\mathrm{m} / \mathrm{z}$ scan range of 400 to 1600 Thomson (Th). Up to the ten most intense ions in MS1 were selected for fragmentation in MS/MS mode. A resolving power of 70,000 full width at half maximum (FWHM), an automatic gain control (AGC) target of $3,000,000$ ions and a maximum ion injection time (IT) of $256 \mathrm{~ms}$ were set for the generation of precursor spectra. To obtain fragmentation spectra, a resolving power of 17,500 FWHM, an AGC target of $1,000,000$ ions and a maximum IT of $64 \mathrm{~ms}$ were used as settings. In order to prevent repeated fragmentation of the most abundant ions, a dynamic exclusion of $10 \mathrm{~s}$ was applied. Ions with one or more than 6 charges were excluded and ions of interest were selected with a 3.0 Th isolation window.

Analysis of the MS/MS data was performed with the PEAKS 7 software (Bioinformatics Solutions Inc., Waterloo, ON, Canada, [22]). Spectra were first selected by quality, and fragmentation spectra with the same mass (less than $5 \mathrm{ppm}$ difference) and retention time were merged. The PEAKS DB function was used to assign the MS/MS spectra to peptide sequences by matching the experimental spectra to theoretical spectra generated from the automatically annotated The Swissprot database and was queried with the taxonomy set to Mus musculus. The following search parameters were used: a precursor mass tolerance of 8 ppm using monoisotopic mass and a fragment mass tolerance of $15 \mathrm{mmu}$. The $-10 \log \mathrm{P}$ score was set at 15 , which resulted in a falsediscovery rate of $0.8 \%$ for peptide spectrum matches. No digestion enzyme was selected. The following post-translation modifications (PTMs) were selected as variable modifications: oxidation (+15.99), acetylation $(+42.01)$, amidation $(-0.98)$, glycine-loss + amidation (-58.01), phosphorylation (+79.97), pyro-glu from E $(-18.01)$, pyro-glu from $Q(-17.03)$, sulfation $(+79.96)$, sodium adduct (+ 21.98) with a maximum of 3 allowed variable PTMs per peptide.

\section{Results}

\subsection{MS imaging reveals learning phase-specific differences}

Based on the cortical and subcortical ROIs illustrated in Fig. 2B, spectra were extracted from DMS and DLS, and peak intensity ratios for $30 \mathrm{~d}$ to $3 \mathrm{~d}$ trained mice were calculated for all ions with a minimum $\mathrm{S} / \mathrm{N}$ of 3 . In DMS and DLS, the peptide with $\mathrm{m} / \mathrm{z}$ of 6724 was detected at a relatively higher intensity in the late learning phase group $(30 \mathrm{~d})$ compared with the early learning phase group ( $3 \mathrm{~d}$ ) (Fig. 2A-C). The peak intensity ratios $(30 \mathrm{~d} / 3 \mathrm{~d})$ for DMS and DLS were 1.24 and 1.36 respectively, clearly demonstrating a differential expression level of $\mathrm{m} / \mathrm{z} 6724$ in these brain regions. When comparing $30 \mathrm{~d}$ with $3 \mathrm{~d}$ trained mice, an apparent relative higher intensity of this ion was also registered in the somatosensory (SS) and motor areas (MO) as well as in two structures of the ventral striatum: the nucleus accumbens (NA) and olfactory tubercle (OT) (Fig. 2A-B). For MO and SS areas separately no obvious differences were found and therefore data for both areas was pooled and ratios were calculated for the entire SS/MO cortical area. Importantly, besides $\mathrm{m} / \mathrm{z} 6724$ we did not observe major changes in peak intensity for any of the other detected peaks in the dataset.

\subsection{Protein of interest matches PEP-19/pcp4}

All fractions obtained from the UPLC fractionation of the peptide extracts were analyzed by MALDI-TOF mass spectrometry and screened for the presence of an ion at $\mathrm{m} / \mathrm{z} 6724$. The mass accuracy window used was \pm 3 Da to accommodate for the inherent low mass accuracy in MALDI MSI, which is the result of the uneven surface (both in thickness and in electrical conductivity) of the tissue. Within this mass window only one relative intense peak could be observed at $\mathrm{m} / \mathrm{z} 6721.3$ in fraction 9 (collected at 4.5 to $5 \mathrm{~min}$ ) and the adjacent fraction 8 . Subsequently, the remainder of fraction 9 was analyzed by LC-MS/MS on the Q-exactive Orbitrap mass spectrometer. An inclusion mass list for precursor selection was used in which the $\mathrm{m} / \mathrm{z}$ values correspond to the precursor mass with 6,7 and 9 charges $(\mathrm{H}+)$ (respectively; 1120.7, 960.9, 840.8). The fragmentation spectrum from $\mathrm{m} / \mathrm{z} 960,75$ yielded the most intense fragments 
A
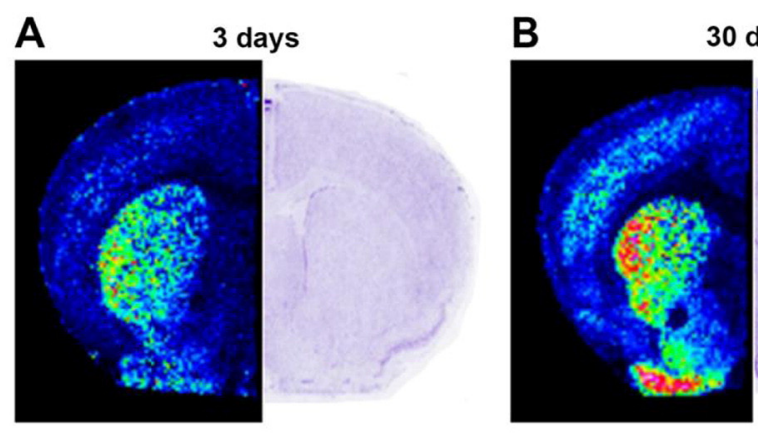

C

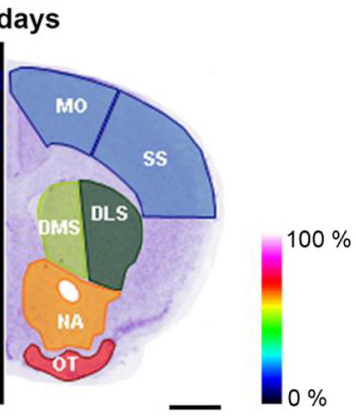

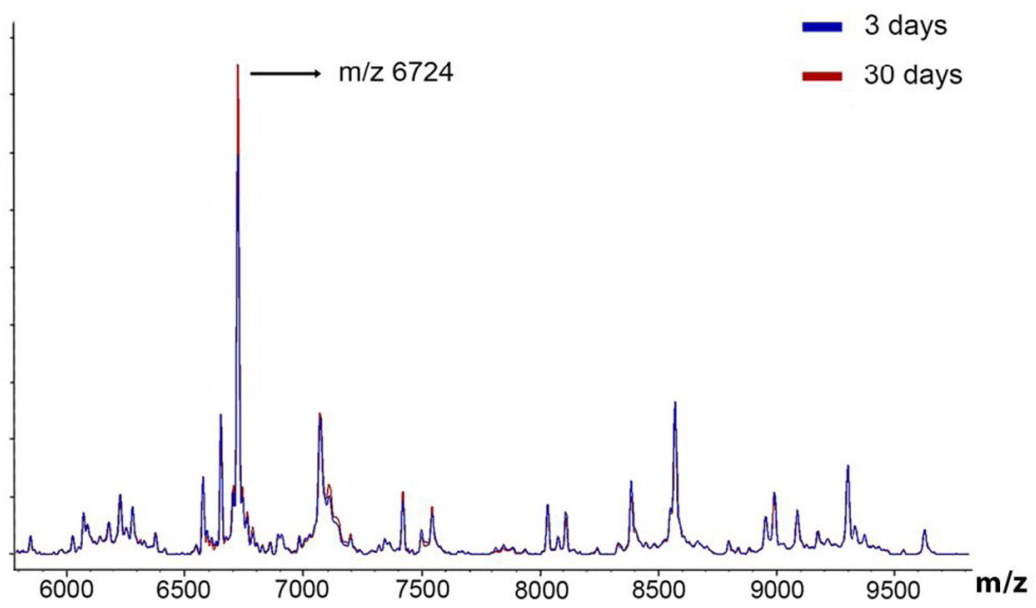

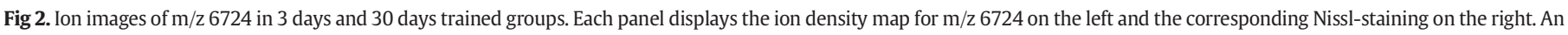

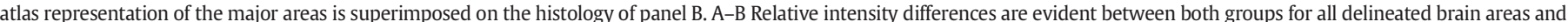

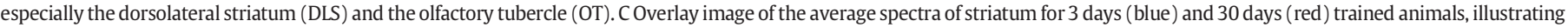
the relative abundance of the masses detected with MSI. Abbreviations: DMS, dorsomedial striatum; SS/MO, somatosensory and motor areas; NA, nucleus accumbens. Scale: 1 mm.

and was used, after deconvolution, for manual de novo sequencing. A clear sequence tag corresponding to the sequence QEEFDI/LDMDA was found. BLAST search against the Mus musculus database revealed $100 \%$ sequence homology with only one protein: PCP4_MOUSE Purkinje cell protein 4 (Total score was 38, E-value 6e-06).

Using Prosight lite more fragments of the spectrum could be annotated for the sequence provided that a $\mathrm{N}$-terminal acetylation was allowed and two de-amidations at probable positions 22 and 53 (both Q). Part of the annotated spectrum is shown in Fig. 3. The full set of all matched fragment ions are shown in Table 1.

\subsection{PEP19/pcp4 mRNA distribution and quantity match MSI findings}

To confirm that PEP-19/pcp4 expression levels are altered between $3 \mathrm{~d}$ and $30 \mathrm{~d}$ of MWM learning, in situ hybridization for PEP19/pcp4 mRNA was performed. Comparison of PEP19/pcp4 gene expression (Allen Brain Atlas, experiment \#79912613) and immunohistochemistry revealed a similar distribution pattern for both mRNA and protein (Fig. 4A-B), which corroborate the MSI ion image data and allowed us to make a concomitant assessment of PEP19/pcp4 differential mRNA expression. A significant upregulation $(P=0.016)$ of $P E P 19 / p c p 4 \mathrm{mRNA}$ was observed in the striatum (DMS + DLS) of $30 \mathrm{~d}$ trained mice compared with the $3 \mathrm{~d}$ trained condition, confirming our MSI findings on the mRNA level (Fig. 4C-D). In addition, when plotting the relative quantity of PEP19/pcp4 mRNA against the escape latency, the data was clearly separated into two groups (Fig. $4 \mathrm{E}$ ), corresponding to the $3 \mathrm{~d}$ trained mice with a lower PEP19/pcp4 expression and longer escape latency compared with the second group containing the $30 \mathrm{~d}$ trained mice with higher PEP19/pcp4 expression. Taken together, these data indicate a clear effect of MWM training on the expression levels of PEP19/pcp4, similar to the data obtained by MSI.

\subsection{PEP-19/pcp4 is present in synaptosomes}

We applied the peptidomics approach for the (semi)-quantitative analysis of neuropeptides in mouse synaptosomes and encountered three fragments of PEP-19 in our dataset. Fig. 5 illustrates how the longest peptide fragment that we detected is icleaved right at the beginning of the IQ-motif. The Cleavage between the AVA-IQS sequences is a predicted calpain cleavage site (predicted by GPS-CCD, [23]). This cleavage is known to give rise to a C-terminal fragment called camstatin.

\section{Discussion}

\section{1. $m / z 6724$ is PEP-19/pcp4}

We demonstrated the validity of MALDI MSI as a powerful tool for neuroscience research, with its (semi)- quantitative analysis providing valuable insights in neurophysiology. The main advantage over classical quantitative proteomics/peptidomics is the information provided on spatial localization of the peptides/proteins in the tissue under investigation. A downside for MSI, however, is that the identification of peptides/proteins of interest remains a problematic challenge [24,25]. Although we previously showed that (very) abundant peptides can be identified via MS/MS directly from tissue [26], this approach cannot be applied to all peptides and proteins due to limitations in mass and 

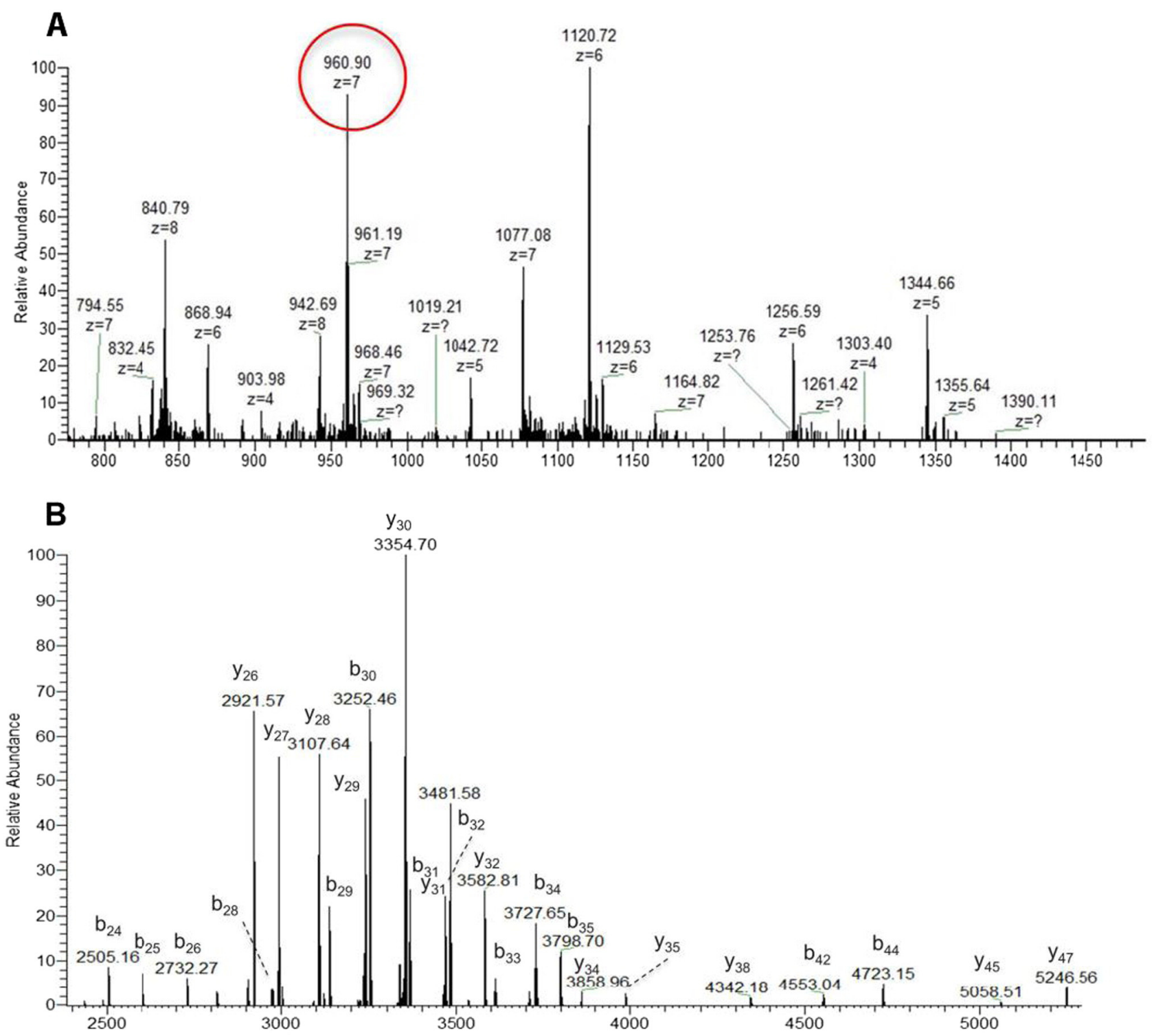

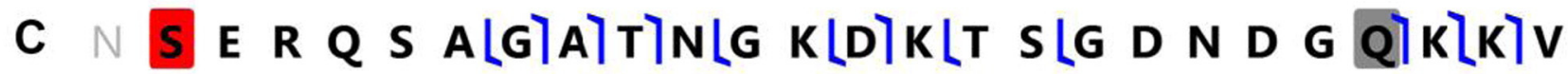

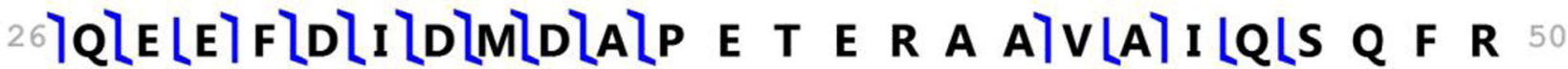 51 K F Q KlKLK A G S Q $\mathbf{S}$}

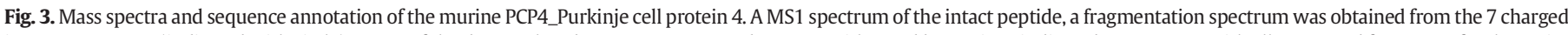

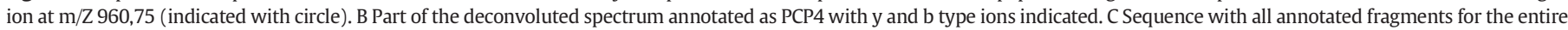
spectrum. Probable location for Acetylation and deamidation are indicated in red and light grey respectively.

concentration. Moreover, interference from other compounds, coisolated with the peptide of interest, usually results in high background further impeding a straightforward identification. These issues can be resolved by micro-extractions straight from the tissue slices and analyzing the extracts by LC-MS/MS either with (proteins) or without (peptides) an intermediate protein digestion step [27]. Alternatively, peptides and proteins can also be identified from whole tissue extracts with LC-MS/MS in which case masses of identified proteins and peptides can be linked with those obtained from the mass spectrometry images [28]. The latter has the advantage that top-down protein identification strategies can be employed in which case identified proteins, with their modification can be linked directly to the obtained images without disrupting the protein digestion protocol [29]. We successfully applied the second strategy. Since most commonly used identification algorithms such as Mascot ${ }^{\mathrm{TM}}$ and Sequest ${ }^{\mathrm{TM}}$ have difficulties with identifying large peptides with multiple charges, the resulting spectra were deconvoluted and manually interpreted. The protein was identified as PCP4_MOUSE Purkinje cell protein 4 (PEP-19/pcp4) based on a sequence tag of 10 amino acids and further confirmed by additional fragment ions in the spectrum.

\subsection{The role of PEP-19/pcp4 in spatial learning and synaptic plasticity}

The MSI approach visualized an increase of $\mathrm{m} / \mathrm{z} 6724$, identified as PEP-19/pcp4, in the striatum of MWM trained mice in the late learning 
Table 1

Full list of matched B and $\mathrm{Y}$ fragment ions.

\begin{tabular}{|c|c|c|c|c|c|c|}
\hline Name & Ion type & Ion number & Theoretical mass & Observed mass & Mass difference (Da) & Mass difference (ppm) \\
\hline B7 & B & 7 & 757.335475 & 757.3338975 & -0.001577467 & -2.082916926 \\
\hline B8 & B & 8 & 828.372585 & 828.3722925 & -0.000292467 & -0.353061998 \\
\hline B9 & B & 9 & 929.420265 & 929.4154505 & -0.004814467 & -5.180075216 \\
\hline B13 & B & 13 & 1343.606555 & 1343.603898 & -0.002657467 & -1.977860907 \\
\hline B22 & B & 22 & 2246.963551 & 2246.959101 & -0.004450467 & -1.980658243 \\
\hline B23 & B & 23 & 2375.058511 & 2375.054107 & -0.004404467 & -1.854466683 \\
\hline B24 & B & 24 & 2503.153471 & 2503.154094 & 0.000622533 & 0.248699541 \\
\hline B25 & B & 25 & 2602.221881 & 2602.2193 & -0.002581467 & -0.992024123 \\
\hline B26 & B & 26 & 2730.280461 & 2730.268431 & -0.012030467 & -4.406311751 \\
\hline B28 & B & 28 & 2988.365641 & 2988.363079 & -0.002562467 & -0.85748104 \\
\hline B29 & B & 29 & 3135.434051 & 3135.433294 & -0.000757467 & -0.241582781 \\
\hline B30 & B & 30 & 3250.460991 & 3250.454671 & -0.006320467 & -1.944483228 \\
\hline B31 & B & 31 & 3363.545051 & 3363.539316 & -0.005735467 & -1.705185093 \\
\hline B32 & B & 32 & 3478.571991 & 3478.56559 & -0.006401467 & -1.840257122 \\
\hline B33 & B & 33 & 3609.612481 & 3609.613417 & 0.000935533 & 0.259178271 \\
\hline B34 & B & 34 & 3724.639421 & 3724.641915 & 0.002493533 & 0.669469669 \\
\hline B35 & B & 35 & 3795.676531 & 3795.668794 & -0.007737467 & -2.038494802 \\
\hline B42 & B & 42 & 4550.037481 & 4550.044167 & 0.006685533 & 1.469335835 \\
\hline B44 & B & 44 & 4720.143001 & 4720.133583 & -0.009418467 & -1.995377445 \\
\hline B54 & B & 54 & 6011.846697 & 6011.826328 & -0.020369467 & -3.388221275 \\
\hline B60 & B & 60 & 6611.185797 & 6611.195372 & 0.009574533 & 1.448232346 \\
\hline Y6 & $\mathrm{Y}$ & 6 & 576.286735 & 576.2855745 & -0.001160467 & -2.013697018 \\
\hline Y7 & Y & 7 & 704.381695 & 704.3800725 & -0.001622467 & -2.303391601 \\
\hline Y15 & $\mathrm{Y}$ & 15 & 1754.942751 & 1754.957909 & 0.015157533 & 8.637052753 \\
\hline Y16 & Y & 16 & 1883.001331 & 1883.017941 & 0.016609533 & 8.820776091 \\
\hline Y18 & $\mathrm{Y}$ & 18 & 2067.122501 & 2067.13195 & 0.009448533 & 4.570862692 \\
\hline Y26 & $\mathrm{Y}$ & 26 & 2920.551861 & 2920.564668 & 0.012806533 & 4.384970283 \\
\hline Y27 & $\mathrm{Y}$ & 27 & 2991.588971 & 2991.606909 & 0.017937533 & 5.995988517 \\
\hline Y28 & Y & 28 & 3106.615911 & 3106.628961 & 0.013049533 & 4.200562121 \\
\hline Y29 & $\mathrm{Y}$ & 29 & 3237.656401 & 3237.669397 & 0.012995533 & 4.013870377 \\
\hline Y30 & $\mathrm{Y}$ & 30 & 3352.683341 & 3352.693843 & 0.010501533 & 3.13227706 \\
\hline Y31 & $\mathrm{Y}$ & 31 & 3465.767401 & 3465.783804 & 0.016402533 & 4.732727624 \\
\hline Y32 & Y & 32 & 3580.794341 & 3580.806256 & 0.011914533 & 3.327343596 \\
\hline Y34 & $\mathrm{Y}$ & 34 & 3856.905341 & 3856.92079 & 0.015448533 & 4.0054219 \\
\hline Y35 & $\mathrm{Y}$ & 35 & 3985.947931 & 3985.947518 & -0.000413467 & -0.10373113 \\
\hline Y38 & Y & 38 & 4341.169881 & 4341.177338 & 0.007456533 & 1.717632187 \\
\hline Y45 & $\mathrm{Y}$ & 45 & 5056.447167 & 5056.479053 & 0.031885533 & 6.305916401 \\
\hline Y47 & $\mathrm{Y}$ & 47 & 5244.526877 & 5244.564569 & 0.037691533 & 7.186831911 \\
\hline Y49 & Y & 49 & 5487.648777 & 5487.685765 & 0.036987533 & 6.740142203 \\
\hline Y51 & $\mathrm{Y}$ & 51 & 5672.765197 & 5672.77876 & 0.013562533 & 2.390815175 \\
\hline Y55 & $\mathrm{Y}$ & 55 & 6015.914377 & 6015.874782 & -0.039595467 & -6.581786974 \\
\hline Y60 & $\mathrm{Y}$ & 60 & 6629.196362 & 6629.191317 & -0.005045467 & -0.761097817 \\
\hline
\end{tabular}

phase compared with mice in the early learning phase and we observed a similar increase in PEP19/pcp4 mRNA. These observations are in accordance with the known role of the striatum in the transitioning of the early, goal-directed learning phase to a late, rigid habituation phase induced by overtraining [2,4], and indicate a role for PEP-19/pcp4 in this process. In addition, both our MSI and ISH findings are in line with the reported predominant presence of PEP-19/pcp4 in mouse and human striatum $[30,31]$.

PEP-19/pcp4, which is a neuron-specific peptide in the adult brain $[30,32]$, binds to the C-domain of calmodulin and attenuates calmodulin-dependent signaling, critical for synaptic function, by modulating $\mathrm{Ca}^{2+}$ binding to calmodulin [32-34]. Wei et al. reported that synaptic plasticity at excitatory synapses is dramatically altered in PEP-19/pcp4 null mice. LTP instead of LTD was induced at parallel fiber Purkinje cell synapses leading to deficits to learn locomotor tasks [12]. Depletion of PEP-19/pcp4 most probably increases the fraction of calcium-bound calmodulin, which in turn affects calcium/CaMdependent protein phosphatases and kinases in favor of LTP [12]. By contrast, increased levels of PEP-19/pcp4 can interfere with the establishment of the calcium/calmodulin complex and could also noncompetitively sequester calmodulin, making it unavailable to calcium/calmodulin-dependent enzymes after calcium concentrations rise [32]. In this study, the analysis of synaptosome content showed that processed forms of PEP-19/pcp4 are present at the presynaps, which again matches the suggestion that PEP-19/pcp4 regulates neurotransmission and synaptic plasticity by modulating calcium/calmodulin signaling. A previous peptidomic study of the murine brain identified an N-terminal fragment of PEP-19 (SERQSAGATNGKDKTSGD), indicating that PEP-19 is indeed cleaved inside neurons [35]. We found three fragments of PEP-19 in mouse brain synaptosomes of which the longest peptide fragment was cleaved right at the beginning of the IQ-motif. The Cleavage between the AVA-IQS sequences is a predicted calpain cleavage site (predicted by GPS-CCD, [23]). This cleavage gives rise to a C-terminal fragment called camstatin, a known blocker of LTP [36].

In the context of MWM learning, the upregulation of PEP-19/pcp4 during the late learning phase was most pronounced in DLS where it could mediate synaptic plasticity processes that facilitate a switch in action control from a more action-outcome guided to a habitual spatial strategy. This is in accordance with the role of DLS in habitual actions previously described $[4,8]$.

Degradation of PEP-19/pcp4 by calpain was already reported by Kanazawa et al. [37] and they related this cleavage of PEP-19/pcp4 to neuronal cell death. Overexpression of PEP-19 resulted in protection against $\mathrm{Ca}^{2+}$ induced cell death, as did inhibition of calpain in normal cells. The protective effect of PEP-19 depends on the Calmodulininteracting IQ motif since interaction inhibiting the mutations in this IQ motif (Q47N/S48T) resulted in the inability of PEP-19/pcp4 to protect against cell death. Moreover, Kanazawa et al. provided preliminary data that this (Q47N/S48T) variant of PEP-19/pcp4 could not be cleaved by calpains (discussion in [37]). This is particularly interesting since the (Q47N/S48T) mutation affects the possible calpain cleavage site that we observed, converting the AVAIQS sequence in AVAINT. The (Q47N/ 
A

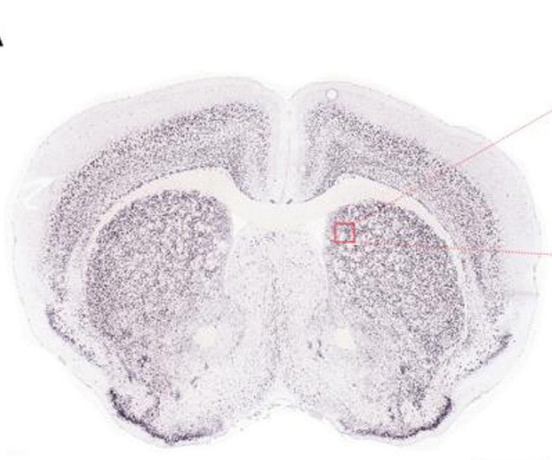

C
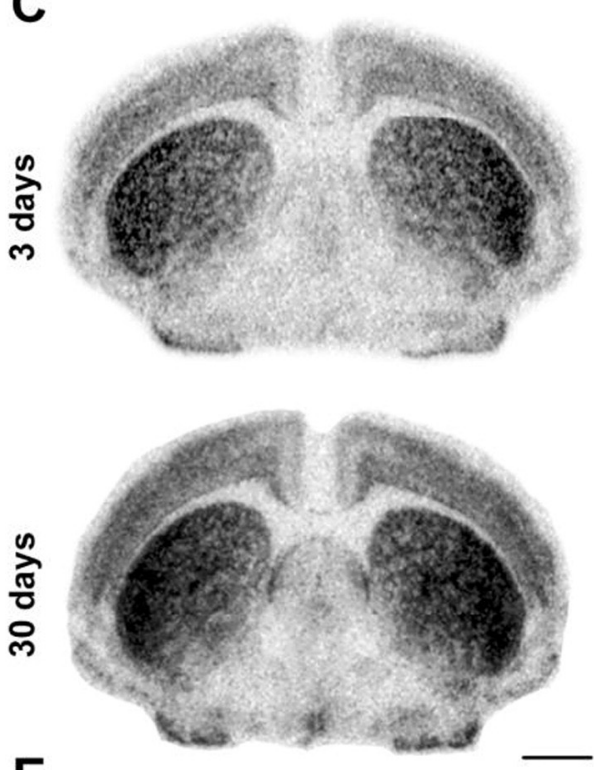

$\mathbf{E}$

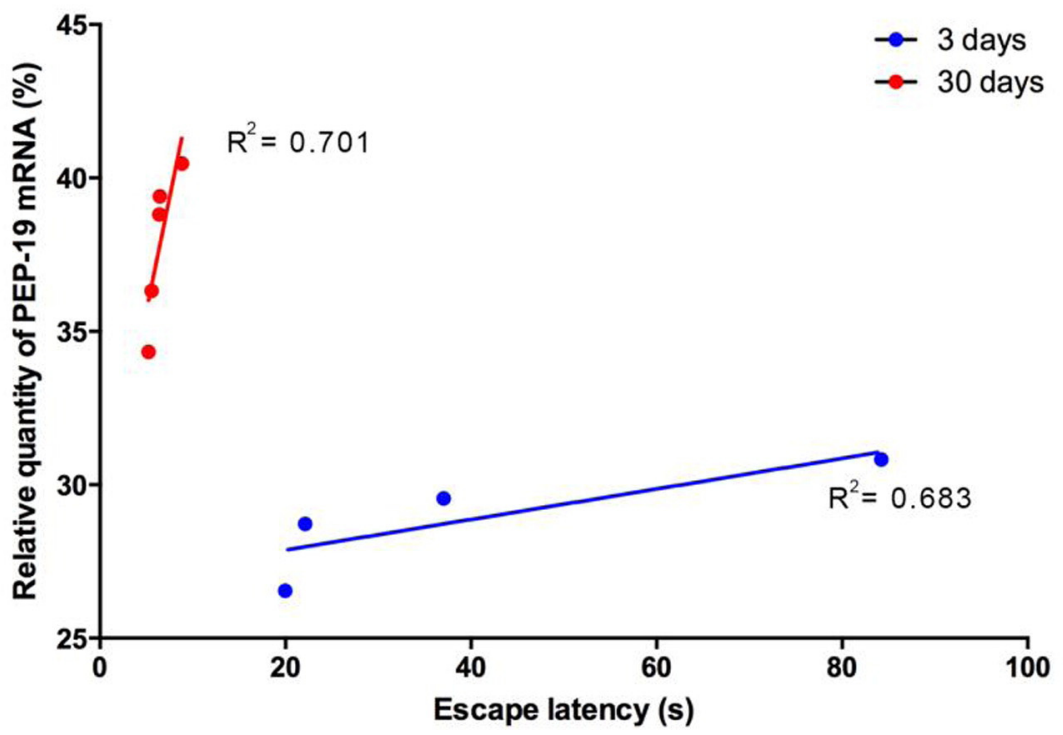

B

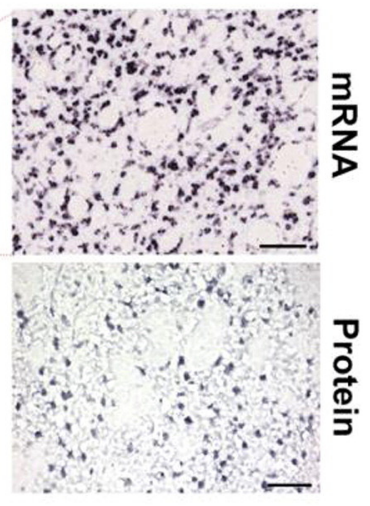

D

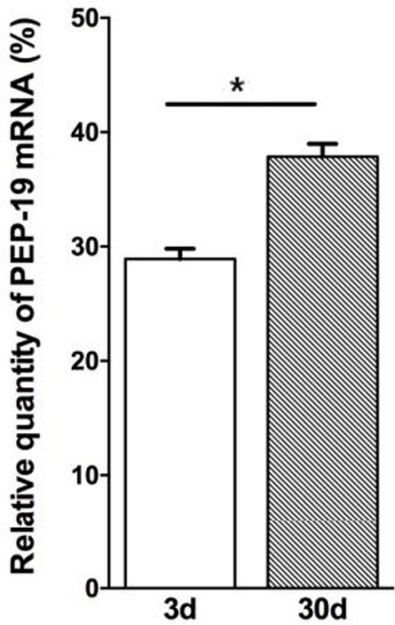

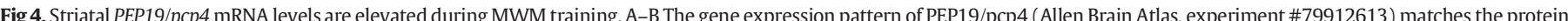

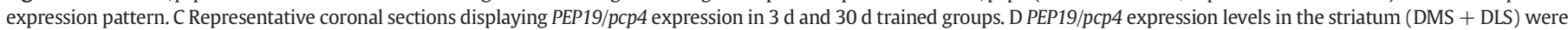

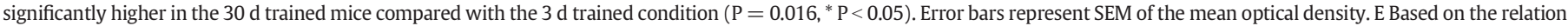

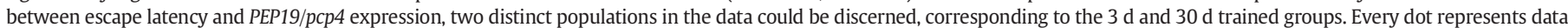
from one animal. 
1 MSERQSAGAT NGKDKTSGDN DGQKKVQEEF DIDMDAPETE RAAVAIQSQF RKFQKKKAGS QS

Fig 5. Illustration of the 3 PEP19/PcP4 peptide fragments identified by the peptidomics strategy applied to mouse brain synaptosomes

S48T) mutant is not predicted to be cleaved by calpain by the GPS-CCD algorithm [23]. Taken together, the peptidomic data indicate that PEP19/pcp4 levels can also be regulated by proteolytic degradation by calpains and that this cleavage results in the inability of PEP-19/pcp4 to interact with calmodulin.

Our observations put PEP-19/pcp4 forward as a regulator of synaptic plasticity in the mouse striatum in the context of spatial learning, thereby generalizing the importance of this small regulator of calmodulin signaling in synaptic plasticity in different brain regions and in the context of different neuronal plasticity phenomena. The outcome of this exploratory study further shows the strategic significance of adding MSI to the state of the art toolbox targeted towards evaluating changes in molecular distribution and expression levels when characterizing the molecular events underlying the response of the nervous system to changes in environmental stimulation.

\section{Conclusions}

Direct comparison of the late learning phase with the early phase revealed one protein of interest with $\mathrm{a} / \mathrm{z}$ of 6724 that was detected at a considerably higher intensity in the DMS and DLS and also displayed higher intensities in the ventral striatum and the somatosensory and motor cortex. This protein was identified as PEP-19/pcp4, which has been previously linked to cerebellar synaptic plasticity and locomotor learning [4,12]. Taken together, our MSI data suggest that PEP-19/ pcp4 could mediate synaptic plasticity in the striatum to facilitate and consolidate habitual actions during the switch of cognitive action control towards the late learning phase.

\section{Funding}

This work was supported by the Research Council of the KU Leuven (GOA/12/008) and the Fund for Scientific Research-Flanders (FWO Vlaanderen) (grant G0D3114N). Jeroen Aerts and Annelies Laeremans were supported by a Ph.D. fellowship of the Agency for Innovation through Science and Technology Flanders (IWT Vlaanderen) and Laurens Minerva by a PhD fellowship of the FWO Vlaanderen.

\section{Conflict of interest statement}

The authors have declared no conflict of interest

\section{Transparency document}

The Transparency document associated with this article can be found, in online version.

\section{Acknowledgements}

We would like to thank Prof. Dr. James I. Morgan of the St. Jude Children's Research Hospital (Memphis, TN) for kindly providing us with the PEP-19/pcp4 antibody, and Lieve Geenen and Karin Schildermans for valuable technical assistance.

\section{References}

[1] R. D'Hooge, P.P. De Deyn, Applications of the Morris water maze in the study of learning and memory, Brain Res. Rev. 36 (2001) 60-90.

[2] D.G. Woolley, A. Laeremans, I. Gantois, D. Mantini, B. Vermaercke, H.P. Op de Beeck, S.P. Swinnen, N. Wenderoth, L. Arckens, R. D'Hooge, Homologous involvement of striatum and prefrontal cortex in rodent and human water maze learning. Proc. Natl. Acad. Sci. U. S. A. 110 (2013) 3131-3136.

[3] A. Laeremans, V. Sabanov, T. Ahmed, J. Nys, B. Plas, K. Vinken, D.G. Woolley, I. Gantois, R. D'Hooge, L. Arckens, D. Balschun, Distinct and simultaneously active plasticity mechanisms in mouse hippocampus during different phases of Morris water maze training, Brain Struct. Funct. (2015) 1-18.

[4] B.W. Balleine, J.P.O.A. Doherty, Human and rodent homologies in action control: corticostriatal determinants of goal-directed and habitual action, Neuropsychopharmacology 35 (2009) 48-69.

[5] H.H. Yin, B.J. Knowlton, The role of the basal ganglia in habit formation, Nat. Rev. Neurosci. 7 (2006) 464-476.

[6] A. Dickinson, B. Balleine, A. Watt, F. Gonzalez, Motivational Control after Extended Instrumental Training, Animal Learning \& ..., 1995.

[7] A.M. Graybiel, Habits, rituals, and the evaluative brain, Annu. Rev. Neurosci. 31 (2008) 359-387.

[8] H.H. Yin, B.J. Knowlton, B.W. Balleine, Lesions of dorsolateral striatum preserve outcome expectancy but disrupt habit formation in instrumental learning, Eur. J. Neurosci. 19 (2004) 181-189.

[9] C.A. Thorn, H. Atallah, M. Howe, A.M. Graybiel, Differential dynamics of activity changes in dorsolateral and dorsomedial striatal loops during learning, Neuron 66 (2010) 781-795.

[10] L. Minerva, A. Ceulemans, G. Baggerman, L. Arckens, MALDI MS imaging as a tool for biomarker discovery: methodological challenges in a clinical setting, Proteomics Clin. Appl. 6 (2012) 581-595.

[11] D.S. Cornett, M.L. Reyzer, P. Chaurand, R.M. Caprioli, MALDI imaging mass spectrometry: molecular snapshots of biochemical systems, Nat. Methods 4 (2007) 828-833.

[12] P. Wei, J.A. Blundon, Y. Rong, S.S. Zakharenko, J.I. Morgan, Impaired locomotor learning and altered cerebellar synaptic plasticity in pep-19/pcp4-Null mice, Mol. Cell. Biol. 31 (2011) 2838-2844.

[13] K. Franklin, G. Paxinos, The Mouse Brain in Stereotaxic Coordinates, 3rd ed. Elsevier, Amsterdam, 2008.

[14] R.T. Fellers, J.B. Greer, B.P. Early, X. Yu, R.D. LeDuc, N.L. Kelleher, P.M. Thomas, ProSight lite: graphical software to analyze top-down mass spectrometry data, Proteomics 15 (2015) 1235-1238.

[15] S.Y. Shu, G. Ju, L.Z. Fan, The glucose oxidase-DAB-nickel method in peroxidase histochemistry of the nervous system, Neurosci. Lett. 85 (1988) 169-171.

[16] E. Van der Gucht, P.R. Hof, L. Van Brussel, K. Burnat, L. Arckens, Neurofilament protein and neuronal activity markers define regional architectonic parcellation in the mouse visual cortex, Cereb. Cortex 17 (2007) 2805-2819.

[17] L. Arckens, F. Zhang, W. Vanduffel, P. Mailleux, J.J. Vanderhaeghen, G.A. Orban, F. Vandesande, Localization of the two protein kinase C beta-mRNA subtypes in cat visual system, J. Chem. Neuroanat. 8 (1995) 117-124.

[18] L. Cnops, T.-T. Hu, J. Vanden Broeck, K. Burnat, G. Van den Bergh, L. Arckens, Ageand experience-dependent expression of Dynamin I and Synaptotagmin I in cat visual system, J. Comp. Neurol. 504 (2007) 254-264.

[19] J. Nys, J. Aerts, E. Ytebrouck, S. Vreysen, A. Laeremans, L. Arckens, The cross-modal aspect of mouse visual cortex plasticity induced by monocular enucleation is age dependent, J. Comp. Neurol. 522 (2014) 950-970.

[20] J. Aerts, J. Nys, L. Moons, T.-T. Hu, L. Arckens, Altered neuronal architecture and plasticity in the visual cortex of adult MMP-3-deficient mice, Brain Struct. Funct. 220 (2015) 2675-2689.

[21] T.M. Wishart, J.M. Paterson, D.M. Short, S. Meredith, K.A. Robertson, C. Sutherland, M.A. Cousin, M.B. Dutia, T.H. Gillingwater, Differential proteomics analysis of synaptic proteins identifies potential cellular targets and protein mediators of synaptic neuroprotection conferred by the slow Wallerian degeneration (Wlds) gene, Mol. Cell. Proteomics 6 (2007) 1318-1330.

[22] J. Zhang, L. Xin, B. Shan, W. Chen, M. Xie, D. Yuen, W. Zhang, Z. Zhang, G.A. Lajoie, B. Ma, PEAKS DB: de novo sequencing assisted database search for sensitive and accurate peptide identification, Mol. Cell. Proteomics 11 (2012), M111.010587M111.010587.

[23] Z. Liu, J. Cao, X. Gao, Q. Ma, J. Ren, Y. Xue, GPS-CCD: a novel computational program for the prediction of calpain cleavage sites, PLoS ONE 6 (2011), e19001.

[24] N.E. Mascini, R.M.A. Heeren, Protein identification in mass-spectrometry imaging, Trends Anal. Chem. 40 (2012) 28-37.

[25] M.M. Gessel, J.L. Norris, R.M. Caprioli, MALDI imaging mass spectrometry: spatial molecular analysis to enable a new age of discovery, J. Proteome 107 (2014) 71-82.

[26] L. Minerva, S. Clerens, G. Baggerman, L. Arckens, Direct profiling and identification of peptide expression differences in the pancreas of control and ob/ob mice by imaging mass spectrometry, Proteomics 8 (2008) 3763-3774

[27] M. Wisztorski, A. Desmons, J. Quanico, B. Fatou, J.-P. Gimeno, J. Franck, M. Salzet, I. Fournier, Spatially-resolved protein surface microsampling from tissue sections using liquid extraction surface analysis, Proteomics (2016).

[28] L. Minerva, K. Boonen, G. Menschaert, B. Landuyt, G. Baggerman, L. Arckens, Linking mass spectrometric imaging and traditional peptidomics: a validation in the obese mouse model, Anal. Chem. 83 (2011) 7682-7691.

[29] J. Sarsby, N.J. Martin, P.F. Lalor, J. Bunch, H.J. Cooper, Top-down and bottom-up identification of proteins by liquid extraction surface analysis mass spectrometry of healthy and diseased human liver tissue, J. Am. Soc. Mass Spectrom. 25 (2014) 1953-1961. 
[30] A.K. Utal, A.L. Stopka, M. Roy, P.D. Coleman, PEP-19 immunohistochemistry defines the basal ganglia and associated structures in the adult human brain, and is dramatically reduced in Huntington's disease, Neurosci. 86 (1998) 1055-1063.

[31] K. Sköld, M. Svensson, A. Nilsson, X. Zhang, K. Nydahl, R.M. Caprioli, P. Svenningsson, P.E. Andrén, Decreased striatal levels of PEP-19 following MPTP lesion in the mouse, J. Proteome Res. 5 (2006) 262-269.

[32] J.R. Slemmon, B. Feng, J.A. Erhardt, Small proteins that modulate calmodulindependent signal transduction: effects of PEP-19, neuromodulin, and neurogranin on enzyme activation and cellular homeostasis, Mol. Neurobiol. 22 (2000) 99-113.

[33] Q.K. Kleerekoper, J.A. Putkey, PEP-19, an intrinsically disordered regulator of calmodulin signaling, J. Biol. Chem. 284 (2009) 7455-7464.

[34] X. Wang, L.W. Xiong, A. El Ayadi, D. Boehning, J.A. Putkey, The calmodulin regulator protein, PEP-19, sensitizes ATP-induced Ca2 + release, J. Biol. Chem. 288 (2013) 2040-2048.
35] M. Fälth, K. Sköld, M. Norrman, M. Svensson, D. Fenyö, P.E. Andrén, SwePep, a database designed for endogenous peptides and mass spectrometry, Mol. Cell. Proteomics 5 (2006) 998-1005.

[36] S.B. Simons, Y. Escobedo, R. Yasuda, S.M. Dudek, Regional differences in hippocampal calcium handling provide a cellular mechanism for limiting plasticity, Proc. Natl. Acad. Sci. U. S. A. 106 (2009) 14080-14084.

[37] Y. Kanazawa, M. Makino, Y. Morishima, K. Yamada, T. Nabeshima, Y. Shirasaki, Degradation of PEP-19, a calmodulin-binding protein, by calpain is implicated in neuronal cell death induced by intracellular Ca2 + overload, Neurosci. 154 (2008) 473-481. 